\title{
Induced pluripotent stem cells: An update
}

\author{
Anubha Singh, Deepak Kumar Singh, Usha Bhoria
}

\begin{abstract}
The discovery that the mature cells can be reprogrammed to become pluripotent increased the enormous interest in induced pluripotent stem cell (iPSC) technology for their potential application in biomedical sciences. The breakthrough research offering induction of pluripotent status in somatic cells by direct reprogramming depends upon the genes used for induction. The induced pluripotent stem cells share features with embryonic stem cells such as pluripotency and immortality. The iPSC technology opened new avenues and provided vast opportunities for regenerative therapy and therapeutic drug development. This review summarizes the developments in iPSC technology highlighting the generation of iPSCs from blood as a source, which is a very important finding, alleviating the need for more invasive techniques such as skin punch biopsies.
\end{abstract}

Anubha Singh ${ }^{1}$, Deepak Kumar Singh ${ }^{2}$, Usha Bhoria ${ }^{3}$ Affiliations: 'Senior Resident, Department of Laboratory Services, Employees' State Insurance Corporation Model Hospital, Ministry of Labor, Noida, Uttar Pradesh, India; 2Junior Specialist, Department of Laboratory Services, Employees' State Insurance Corporation Model Hospital, Ministry of Labor, Noida, Uttar Pradesh, India; ${ }^{3}$ Senior Specialist, Department of Laboratory Services, Employees' State Insurance Corporation Model Hospital, Ministry of labor, Noida, Uttar Pradesh, India.

Corresponding Author: Anubha Singh, Senior Resident, Department of Laboratory Services, Employees' State Insurance Corporation Model Hospital, Noida, Uttar Pradesh, India; E-mail: anubha.singh0111@gmail.com

Received: 11 January 2015

Accepted: 26 February 2015

Published: 09 March 2015
Keywords: Induced pluripotent stem cell (iPSC), Reprogramming, Hematopoietic cells, Peripheral blood

\section{How to cite this article}

Singh A, Singh DK, Bhoria U. Induced pluripotent stem cells: An update. Int $\mathrm{J}$ Blood Transfus Immunohematol 2015;5:6-13.

Article ID: 100016IJBTIAS2015

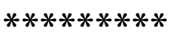

doi:10.5348/ijbti-2015-16-RA-2

\section{INTRODUCTION}

Transplantation of healthy functional cells which can repair or replace the damage through the process of regeneration is likely to provide a cure for diseases like loss of immune cells, burns, fractures, strokes, renal failure and cancer. Embryonic stem (ES) cells derived from the inner cell mass of mammalian blastocysts from IVF clinic embryos in 1998 by Dr. Thomson and colleagues have the ability to grow indefinitely while maintaining the pluripotency [1]. Use of human embryos, however, faces moral and ethical issues. In addition, it is difficult to generate patient-or disease-specific ES cells, which are required for their effective application.

One way to circumvent these issues is to induce pluripotent status in somatic cells by direct reprogramming. In the field of regenerative medicine, induced pluripotent cells hold great promise as they can be generated from adult cells and propagate indefinitely, giving rise to every other cell type in the body such as heart, pancreatic, neurons, and liver cells. Pluripotent cells represent themselves as a single source of cells that 
have the ability to replace the tissues and organs that are lost due to damage or disease [2].

\section{FIRST GENERATION MOUSE INDUCED PLURIPOTENT STEM CELLS (iPSCS)}

Mature cells from a person's body contain dormant genes that are associated with pluripotency --the ability to become any of the 220 cell types in a human body. Yamanaka and colleagues in 2006, developed first generation "induced pluripotent stem cells (iPSCs)" from mouse embryonic fibroblasts (MEF) and adult mouse tailtip fibroblasts by the retrovirus-mediated transfection of four transcription factors, namely Oct3/4, Sox2, c-Myc and Klf4 $[2,3]$ The first generation mouse induced pluripotent stem cells (mouse iPSC) are indistinguishable from ES cells in morphology, proliferation and gene expression.

To generate the iPSCs, twenty-four genes which were previously identified as important in embryonic stem cells were chosen. Retroviruses were used to deliver these chosen genes into fibroblasts from mice. Through antibiotic selection, engineered mouse fibroblasts cells that reactivated the ESC-specific gene, Fbx15, were isolated. Colonies of such cells resembled embryonic stem cells that have the ability to propagate indefinitely. Yamanaka and colleagues further narrowed down the induction factors from the initial pool of 24, finally identifying the four factors, Oct4, Sox2, cMyc, and Klf4, which are sufficient as well as necessary to generate the iPSCs.

These were the first generation iPSCs which showed unlimited potential for self-renewal and properties similar to ES cells; demonstrating pluripotency by contributing to lineages from all three germ layers. These iPSCs had gene expression and epigenetic marks, somewhere in between those of fibroblasts and an ES cells. When injected into developing embryos, these cells failed to produce viable chimeras.

\section{SECOND GENERATION MOUSE IN- DUCED PLURIPOTENT STEM CELLS}

Yamanaka and colleagues again in June 2007, successfully reprogrammed mouse fibroblasts into iPS cells. When transplanted into blastocysts, mouse iPS cells gave rise to adult chimeras, which are competent for germline transmission. These results are proof of principle that pluripotent stem cells can be generated from somatic cells by the combination of a small number of factors. These iPSCs were derived from mouse fibroblasts by retroviral-mediated expression of the same four transcription factors (Oct4, Sox2, c-Myc, Klf4). The marker for selection of pluripotent cells used was Nanog, instead of Fbx15 used in previous studies. Nanog, is a gene that is functionally important in ESCs. This strategy created iPSCs that were more similar to ESCs than the first generation of iPS cells. This further proved that it was possible to create iPSCs that are functionally identical to ESCs [4]. Studies found mouse embryonic fibroblast (MEF)-derived iPS cells that could be injected into tetraploid blastocysts and resulted in the live birth of mice derived entirely from iPS cells, thus ending the debate over the equivalence of embryonic stem cells (ESCs) and iPS with regard to pluripotency.

One pitfall observed while generating iPSCs was that $20 \%$ of the chimeric mice developed cancer. This phenomenon was later identified due to two of the four genes used (c-Myc and Klf4) which are oncogenic. Later reports by work of Yamanaka revealed that c-Myc was not necessary to create iPSC, however, the new protocol without using c-Myc was not very efficient and required long time. The advantage was that the cancer did not develop by the resulting chimeras [4]. Various cells such as skin cells, prostate cells, liver, blood cells, adult stomach and urinary tract cells have been induced to generate the induced pluripotent cells [5].

\section{HUMAN INDUCED PLURIPOTENT STEM CELLS - A BREAKTHROUGH RESEARCH IN MODERN MEDICAL SCIENCE}

In a breakthrough research in November 2007, iPSCs were created from adult human cells [6,7]. Another group, Thomson and colleagues used Oct3/4, Sox2, Nanog, Lin28 genes, however they used a lentiviral system [8]. Previously Yamanaka and colleagues had successfully generated induced pluripotent stem cells from human fibroblast cells using Oct3/4, Sox2, Klf4, and c-Myc with transformation done using a retroviral system [9].

In November 2012, human iPSCs were generated from exfoliated renal epithelial cells present in urine [10]. This method of acquiring donor cells was comparatively less invasive and simple. The induction procedure was less time consuming. Urinary cell culture took about two weeks and further reprogramming and higher yield took $3^{-4}$ weeks. Renal epithelial cells used to generate the urinary iPSCs represent a promising choice as they are readily available using non-invasive methods, and show good differentiation potential ability. This technology can be highly successful in understanding mechanisms, disease models and effective treatment of patients with genetic diseases including those affecting kidney functions.

\section{GENERATION OF IPS CELLS DEPENDS ON THE GENES USED FOR THE IN- DUCTION}

Induction of pluripotent status in somatic cells by direct reprogramming depends upon the genes used for induction. There are certain transcriptional regulators 
which are involved in the induction process which leads to ESCs-like colonies.

A successful induction is possible by factors which include Oct-3/4 and certain members of the Sox gene family such as Sox2, Sox1, Sox3, Sox15 and Sox18. They are extremely crucial as their absence makes induction impossible. For maintaining the pluripotency, Oct-3/4 which is one of the family of octamer has been identified as important transcription factor. Its expression has been exclusively found in pluripotent stem cells. Similar to Oct-3/4, Sox family of genes is associated with maintaining the pluripotency, though limited to multipotent and unipotent stem cells. Sox1 has the same induction efficiency as Sox2, whereas other genes such as Sox3, Sox15 and Sox18 also yield iPSC though with low efficiency.

The efficiency of induction is increased by many additional genes which have been identified as members of the Klf family such as Klf1, Klf2, Klf4, and Klf5; the Myc family such as c-myc, L-myc, and N-myc; Nanog, and Lin28. Yamanaka et al. and other researchers identified Klf4 of the Klf family of genes, for generation of mouse and human iPSC. Another family of genes which Yamanaka et. al. and other researchers demonstrated as important in the generation of mouse and later human iPSCs were Myc family of genes. These include c-Myc, $\mathrm{N}-\mathrm{Myc}$ and L-Myc. However, due to the fact that Myc family of genes are proto-oncogenes and are implicated in cancer, halted the usage of c-Myc in induction process and eventually in clinical therapies. Eliminating Myc gene lowers the efficiency by 100-fold. During the study $20 \%$ mice developed lethal teratomas after transplantation with c-myc-induced iPS cells. N-myc and L-myc have been identified to induce with similar efficiency as c-Myc. Along with Oct-3/4 and Sox2, Nanog has been identified as important in promotion of pluripotency in ESC. Thomson et. al, generated iPSC using Nanog as one of the factors, though Yamanaka et al., reported it to be unnecessary for induction. Another factor which Thomson et al. used in generation of iPSC was Lin28, although in his report he mentioned it as unnecessary. Lin28 is an mRNA binding protein [40] expressed in embryonic stem cells and embryonic carcinoma cells associated with differentiation and proliferation. Glis1 is transcription factor that can be used with Oct-3/4, Sox2 and Klf4 to induce pluripotency. It poses numerous advantages when used instead of C-myc [11].

\section{SAFETY ISSUES FOR REGENERATIVE MEDICINE}

Human iPSCs provide promising opportunities in biomedical technology, cell therapies for regenerative medicine and stem cell modelling of human diseases and drug development. On the other hand, recent reports have emphasized the pitfalls of iPSC technology, including the potential for genetic and epigenetic abnormalities, tumorigenicity, and immunogenicity of transplanted cells. The enormous benefit associated with iPSCs technology in conditions where there is no current possible treatment outweighs the pitfalls and drawbacks.

Yamanaka and colleagues observed one pitfall while generating iPSCs was that $20 \%$ of the chimeric mice developed lethal teratomas. The propensity of iPSC to form tumors is the major concern with their potential clinical application. [12] iPSCs are generally predicted to be less safe and highly tumorigenic than hESC, as they are generated with high efficiency using modifications. Members of the Myc family including c-Myc are identified as proto-oncogenes and used to generate iPSCs. Though later it was not found to be necessary for iPSC generation, its omission leads to lowering of induction efficiency upto 100 fold. Research to identify many other genes that have been shown to generate iPSC revealed that they are linked to tumor formation.

Zhou et al., in 2011 expressed a concern regarding the immunogenicity of iPSCs. He conducted a study involving a teratoma formation assay and showed that the immune response produced by iPSCs was strong enough to cause rejection of the cells. He then performed a similar procedure on genetically equivalent ESCs. In this study teratomas were found, which indicated that the cells were tolerated by the immune system [13]. Araki et al. in 2013, in order to reproduce the conclusion obtained by Zhou et al. used a different approach where they took cells from a chimera that had been grown from iPSC clones and a mouse embryo and transplanted into syngenic mice. They then performed a similar test using ESCs instead of iPSC clone and compared the results. Their results demonstrated no significant difference in the immunogenic response produced by the iPSCs and the ESCs. Additionally little or no immunogenic response was reported for both cell lines [14].

A crucial area for future research in the field of iPSC is directly testing iPSC tumor-forming ability using methods and protocols that mimic the approaches that would be used for regenerative therapy [15]. When injected into immunodeficient mice, iPSCs form teratoma, much the same way as ESC. FDA considered teratoma formation as a major obstacle to stem-cell based regenerative medicine. Concerns over generation of safe iPSCs leads to newer methods which involves recombinant proteins, however they show very low efficiency. Protocols for conversion of somatic cells to safe iPSC require refinements and better approaches for carrying out induction. Many different approaches such as using adenovirus or plasmids are generally thought to be safer than retroviral methods.

A recent study showed the clinical application of iPSC on motor functional recovery after spinal cord injury. When human iPSCs were transplanted into the mice with spinal cord injuries, the cells showed the ability to differentiate into three neural lineages in the spinal cord. The cells were shown to stimulate regrowth of the damaged spinal cord, maintained myelination, and formed synapses. No tumor formation was observed and 
these positive outcomes were consistently observed for over 112 days after the spinal cord injury [16].

\section{CHALLENGES IN DIRECT REPRO- GRAMMING CELLS TO PLURIPOTENCY}

Successful generation of induced pluripotent stem cells from human fibroblast cells by Yamanaka et al. has shown the way for exploring more stem-cell based regenerative medicine, however, there are still challenges associated with the methods of induction of pluripotent status in somatic cells by direct reprogramming. Major concerns lie with the low efficiency, tumor formation and successfully reaching the goal of the protocol eventually leading to clinical applications.

Tumor formation: One of the biggest challenges is tumor formation. Though the role of transcription factors in successful induction of iPSC has been established, this comes with a threat to tumor formation, limiting the applications in clinical therapies. Some of the reprogramming factors are oncogenes, which bring a potential risk of tumor formation. Identifying transcription factors not associated with oncogenic potential is required. Studies show that deletion or inactivation of tumor suppressor p53 which is a master regulator of cancer, markedly increases the reprogramming efficiency [17]. Thus there seems to be a tradeoff between reprogramming efficiency and tumor generation.

Low efficiency: In Yamanaka's initial mouse study the rate at which the somatic cells were reprogrammed into iPSCs was 0.01-0.1\%. Therefore the efficiency of conversion of somatic cells to iPSCs has been extremely low [1]. The low efficiency rate may reflect the need for precise timing, balance, and absolute levels of expression of the reprogramming genes. It may also suggest a need for rare genetic and/or epigenetic changes in the original somatic cell population or in the prolonged culture. Luo et al, in 2013, reported that for efficient reprogramming, the downregulation of the nucleosome remodeling and deacetylation (NuRD) complex is required. It has been shown that overexpression of Mbd3, inhibits induction of iPSCs by establishing heterochromatic features and silencing embryonic stem cell-specific marker genes, including Oct4 and Nanog. $\mathrm{Mbd} 3$ is a core member of the $\mathrm{Mbd} 3 / \mathrm{NuRD}$ (nucleosome remodelling and deacetylation) repressor complex. When Mbd3 is depleted, reprogramming efficiency improves, facilitating the generation of iPSCs. Mbd3/NuRD has been found to be an important epigenetic regulator that restricts the expression of key pluripotency genes suggesting that drug-induced down regulation of $\mathrm{Mbd} 3 /$ NuRD may be a powerful means to improve the efficiency and fidelity of reprogramming [18]. Rais et al, in 2013 showed that the depletion of Mbd3, together with Oct4, Sox2, Klf4 and Myc transduction and reprogramming in naive pluripotency promoting conditions, resulted in deterministic and synchronized iPSC reprogramming (near 100\% efficiency within seven days from mouse and human cells) [19].

Genomic Insertion: Genome integration of the transcription factors is a challenge in reprogramming. This poses a risk of mutations being inserted into the target cell's genome [20]. Plasmids, adenoviruses, and transposon vectors have been explored as a strategy for avoiding genomic insertion but they have been associated with lower throughput.

Incomplete reprogramming: Achieving complete reprogramming is also a challenge. The genome-wide epigenetic code must be reformatted to that of the target cell type in order to fully reprogram a cell [21].

\section{APPLICATIONS OF iPSCS IN MEDICAL RESEARCH}

ESCs have ethical issues related to their utility which is overcome by patient-specific iPSCs. Patient-specific iPSCs also bypass the possible immune rejection. Therefore, iPSCs have been considered superior in regenerative medicine for stem cell modelling of human diseases, organ and tissue regeneration and screening of pharmacological compounds for drug development.

\section{Regenerative medicine}

Scientists have successfully reprogrammed several somatic cell types, including fibroblasts and, keratinocytes, extraembryonic tissues, umbilical cord blood mononuclear cells (UCBMCs) and peripheral blood cells and cells obtained from urine to iPSCs. Several factors, including the age, origin and type of the cells used, deeply impact the reprogramming efficiency and the quality of the iPSCs generated.

\section{Disease modelling}

Induced pluripotent stem cells are endowed with unique features of self-renewal and pluripotency, which provide an unlimited source of patient-specific cells with the ability to generate any type of cell in the body. The iPSCs have an important application in understanding the molecular and cellular basis of human diseases, as they are derived from adult patients. Patient-derived iPSCs exhibit cellular defects which are not observed in iPSCs derived from normal healthy individuals. This property provides extremely unique insights into the pathophysiology of the disease. The iPSCs have been generated for a wide variety of human genetic diseases, including common disorders such as Down's syndrome and polycystic kidney disease [22, 23].

Methods used to generate the iPSCs are critical for regenerative medicine. Originally, retroviral or lentiviral systems were used to generate the first human iPSCs. Their insertions in the genome could give rise to severe damage to the induced cells. However, iPSCs can now be 
generated without integration of transgenes. These iPSCs are more suitable for clinical applications. Additionally, manipulations of the culture conditions used for the iPSCs, including the use of Xeno-Free culture systems, also should be evaluated in more depth for transplantation.

\section{Organ regeneration}

Many groups made efforts for organ regeneration using iPSC technology and they have been successful in their achieving their goals. Some successful organ regeneration examples are mentioned.

A successful use of iPSCs in organ regeneration was made in obtaining vascularized and functional human livers generated in vivo by transplantation of liver buds made in vitro [24]. This was a proof-of-concept of using iPSCs to generate human organ. A mixture of three different stem cells was used to grow human 'liver buds' (iPSC-LBs): hepatocytes (for liver function) coaxed from iPSCs; endothelial stem cells (to form lining of blood vessels) from umbilical cord blood; and mesenchymal stem cells (to form connective tissue). This new concept similar to the process in fetal development, allowed different cell types to self-organize into a complex organ. After growing in vitro for a few days, the liver buds were transplanted into mice where the 'liver' quickly connected with the host blood vessels and continued to grow. An important finding was that it performed regular liver functions which include metabolizing drugs and producing liver-specific proteins. With this success, it paves the way to explore more into longevity monitoring of the transplanted organ in the host body, their ability to integrate or avoid rejection and whether they transform into tumors.

Rat pancreases were successfully generated by iPSC technology in Mouse body. Because of pancreatic insufficiency, Pdx1 deficient mice die after birth. Pdx1 -/mouse blastocyst were then injected with Rat pluripotent stem cells to rescue them. The rescued mouse had fully functional pancreas derived from rat pluripotent stem cells [25].

The $3-\mathrm{D}$ structures of the retina and pituitary gland were generated by self-organization of pluripotent stem cells in vitro [26]. The unique combination of $3-\mathrm{D}$ differentiation and improved efficiency systems for iPSC induction will be important in provide new insights and strategies for regenerative therapy.

\section{Repair of damaged tissues}

Another important application where the iPSCs have been successfully used is tissue repair and regeneration. Using plasmid DNA, embryonic cord-blood cells were induced into pluripotent stem. Vascular progenitor', the high-quality, multipotent vascular stem cells were identified using cell surface endothelial/pericytic markers CD31 and CD146. Directly injecting the iPSCs into the vitreous of the damaged retina of mice, the stem cells which were engrafted into the retina, successfully grew and repaired the damaged vascular vessels [27].
In another study, labeled iPSCs-derived NSCs were implanted into TBI rats and SCI monkeys, one week after injury, showed obvious motor function improvement in transplanted animals. The studies from this finding provide the necessary foundation for future clinical application of iPSCs for CNS injury [28].

\section{Generation of red blood cells}

Another important clinical application of iPSC technology is the synthesis of type O red blood cells. In a step-wise manner the cells were first induced to become a mesoderm and then blood cells and at the end red blood cells. The important application of this synthetic generation of type $\mathrm{O}$ red blood cells is that type $\mathrm{O}$ blood can be universally transfused to all the patients. These were initial reports of human transfusion which will take time to reach the clinical setting [29].

\section{Therapeutic Drug development}

One of the most promising application of iPSCs reside in screening of chemical compounds and natural derivatives to identify useful therapeutic drug candidates. Many studies have published the disease modeling and drug screening using patient-derived iPSCs. In one study, it was shown that anacardic acid has the ability to diminish the phenotype in a disease model of amyotrophic lateral sclerosis ALS using patient-specific iPS cells [30]. A project named, StemBANCC targets to generate and characterize 1500 high quality human iPSC lines from 500 people that will be utilized to study a spectrum of diseases and test for drug efficacy and safety. Skin samples from normal healthy individuals, patients with specific diseases and people who exhibit adverse drug reactions will be obtained.

\section{CLINICAL TRIAL WITH INDUCED PLURIPOTENT STEM CELLS}

iPSCs Implanted into Humans in Japan: Initially when iPSCs technology was described by Yamanaka et al., many research groups focused on creating iPSCs from adult cells. The earlier clinical trials involved creation of iPSC lines from specific patient segments and further evaluating these cell lines for purpose of studying the model for human diseases within that patient segment. Currently there are many ongoing clinical trials which involve understanding the molecular mechanisms, phenotypes, early risk assessments of many human disorders including cardiovascular diseases, COPD, eye diseases, spinal cord injury, and many psychiatric disorders. Currently, there are more than 39 different clinical trials underway which are listed at the ClinicalTrials.gov website.

The first ever human clinical trial using autologous iPSCs was approved in Japan at the Riken Center for Developmental Biology. The autologous iPSCs alleviate 
the need for ESCs and are assumed to pose no risk of immune rejection [31]. The trial involved reprogramming of wet age-related macular degeneration patient's skin cells, producing pluripotent stem cells from them, then differentiating them into retinal pigment epithelial (RPE) cells. The RPE layer of the eye supports the more complex networks of light-converting photoreceptors. The cell sheet was transplanted into the affected retina where the degenerated RPE tissue has been excised. The postoperation safety and vision restoration monitoring of the cell recipient's condition will be carried out by the team which is expected to last 1-3 years [32].

Other iPSC projects reported to be planned: Jun Takahashi at CiRA planned a Parkinson's iPSCs trial, where he plans to give dopaminergic neurons from iPSCs to Parkinson's patients in two to three years. Experiments to optimize condition for neural induction from human iPS cells, and confirmation of safety profile in the primate model is ongoing [33].

Hideo Okano, a neural stem cell researcher at Keio University School of Medicine, has planned a iPSC spinal cord injury trial. He has focused on investigating the development of cell-based therapy for injured spinal cord using iPS cells-derived neural stem/ progenitor cells [34]. They have also successfully induced the differentiation of mouse and human iPSCs toward neural stem cells [35] and transplanted them into mouse and non-human primate spinal cord injury models [36]. Their results demonstrated that transplanted cells differentiated into neurons, astrocytes and oligodendrocytes, and transplantation of these cells resulted in functional recovery without tumor formation upon selection of appropriate cell lines.

Koji Eto, a blood stem cell researcher at CiRA, headed the preclinical in vivo and ex vivo studies focused on the length of time iMKPCs could be established prior to transfusion, while retaining their functional abilities and use of hiPSCs as a powerful research tool, with the potential of novel targeted therapeutics for hematopoietic disorders.

An international collaborated project, StemBANCC, was formed in 2012 to build a collection of iPS cell lines for drug screening for a variety of disease. Managed by the University of Oxford, the effort pooled funds and resources from 10 pharmaceutical companies and 23 universities. The goal is to generate a library of 1,500 iPS cell lines which will be used in early drug testing by providing a simulated human disease environment [36]. In this project the raw materials for the project are mostly skin samples taken from patients with certain specific diseases, individuals who display ADRs, and normal healthy individuals. Individual's informed consent is important prior to sample collection and protocols are in line with strict ethical standards.

Inducing pluripotency in peripheral blood An advantage over other methods: The iPSCs are generated by a process which involves the conversion of ordinary cells, like skin cells, so that their properties simulate those of embryonic stem cells. Along with skin and other tissue cells and blood cells can also be used to create iPSCs. Induced pluripotent stem cells (iPSCs) have become important cell sources for genetic disease models, and they have the potential to be cell sources for future clinical therapies. However, invasive tissue sampling reduces the number of candidates who consent to donate cells for iPSC generation. In addition, integrated transgenes can potentially insert at inappropriate points in the genome, and in turn have a direct oncogenic effect. Technical modifications using a combination of activated T cells and a temperature-sensitive mutant of Sendai virus (SeV) can avoid invasive tissue sampling and residual transgene issues in generating iPSCs. Such advances may increase the number of consenting patients for cell donations [37].

Using blood as a cell source of iPSCs cells has two major advantages, one is the blood is the easiest, most accessible source of cells, and second blood collection and storage is a well-established part of the medical system [38]. Among all the blood cells, only white blood cells ( $\mathrm{T}$ cells and few myeloid cells) can be converted to iPS cells. Patient-specific iPS cells could be generated for the "customized" transplantation medicine to treat various disorders. Similarly, disease-specific iPSCs could be used to study the pathogenesis and test drugs. Recently, it was reported that using autologous iPS cells, mice with sickle cell anemia were treated. This is the first direct evidence for clinical promises of iPS cells, and it provided people with hope and confidence for further studies and eventually, therapeutic applications [39].

Peripheral blood-derived human iPSC lines are comparable to human ESCs with respect to morphology, expression of surface antigens, activation of endogenous pluripotency genes, DNA methylation and differentiation potential [39].

\section{CONCLUSIONS}

Human induced pluripotent stem cells hold great promise for regenerative medicine. It is only eight years since the naissance of iPS cells in mouse. Since then methods for iPS cell generation have been refined a lot and human iPS cells have been successfully established. iPS cells have shown as a promising treatment modality for various diseases. With rapid progress in techniques, iPS cell research will undoubtedly become one of the leading studies in life sciences and great improvements in iPS cell research would exceed our current imagination.

$$
* * * * * * * * *
$$

\section{Author Contributions}

Anubha Singh - Substantial contributions to conception and design, Acquisition of data, Analysis and interpretation of data, Drafting the article, Revising it critically for important intellectual content, Final approval of the version to be published 
Deepak Kumar Singh - Analysis and interpretation of data, Revising it critically for important intellectual content, Final approval of the version to be published Usha Bhoria - Analysis and interpretation of data, Revising it critically for important intellectual content, Final approval of the version to be published

\section{Guarantor}

The corresponding author is the guarantor of submission.

\section{Conflict of Interest}

Authors declare no conflict of interest.

\section{Copyright}

(C) 2015 Anubha Singh et al. This article is distributed under the terms of Creative Commons Attribution License which permits unrestricted use, distribution and reproduction in any medium provided the original author(s) and original publisher are properly credited. Please see the copyright policy on the journal website for more information

\section{REFERENCES}

1. Thomson JA, Itskovitz-Eldor J, Shapiro SS, et al. Embryonic stem cell lines derived from human blastocysts. Science 1998 Nov 6;282(5391):1145-7.

2. Takahashi K, Yamanaka S. Induction of pluripotent stem cells from mouse embryonic and adult fibroblast cultures by defined factors. Cell $2006 \mathrm{Aug}$ 25;126(4):663-76.

3. The Nobel Prize in Physiology or Medicine-2012 Press Release. Nobel Media AB. 8 October 2012.

4. Wernig M, Meissner A, Foreman R, et al. In vitro reprogramming of fibroblasts into a pluripotent EScell-like state. Nature 2007 Jul 19;448(7151):318-24.

5. Moad M, Pal D, Hepburn AC, et al. A novel model of urinary tract differentiation, tissue regeneration, and disease: reprogramming human prostate and bladder cells into induced pluripotent stem cells. Eur Urol 2013 Nov;64(5):753-61.

6. Baker M. Adult cells reprogrammed to pluripotency, without tumors. Nature Reports Stem Cells. 2007.124 Available at: http://www. nature.com/stemcells/2007/0712/071206/full/ stemcells.2007.124.html Retrieved 2007-12-11 doi:10.1038/stemcells.2007.124.

7. Kolata G. Scientists Bypass Need for Embryo to Get Stem Cells. The New York Times. 2007. Available at: http://www.nytimes.com/2007/11/21/ science/21stem.html?_r=1\& Retrieved 2007-12-11

8. $\mathrm{Yu}$ J, Vodyanik MA, Smuga-Otto K, et al. Induced pluripotent stem cell lines derived from human somatic cells. Science 2007 Dec 21;318(5858):191720.

9. Takahashi K, Tanabe K, Ohnuki M, et al. Induction of pluripotent stem cells from adult human fibroblasts by defined factors. Cell 2007 Nov 30;131(5):861-72.

10. Zhou T, Benda C, Dunzinger S, et al. Generation of human induced pluripotent stem cells from urine samples. Nat Protoc 2012 Dec;7(12):2080-9.
11. Maekawa M, Yamaguchi K, Nakamura T, et al. Direct reprogramming of somatic cells is promoted by maternal transcription factor Glis1. Nature 2011 Jun 8;474(7350):225-9.

12. Knoepfler PS. Deconstructing stem cell tumorigenicity: a roadmap to safe regenerative medicine. Stem Cells 2009 May;27(5):1050-6.

13. Zhao T, Zhang ZN, Rong Z, Xu Y. Immunogenicity of induced pluripotent stem cells. Nature 2011 May 13;474(7350):212-5.

14. Araki R, Uda M, Hoki Y, Negligible immunogenicity of terminally differentiated cells derived from induced pluripotent or embryonic stem cells. Nature $2013 \mathrm{Feb}$ 7;494(7435):100-4.

15. Gutierrez-Aranda I, Ramos-Mejia V, Bueno C, et al. Human induced pluripotent stem cells develop teratoma more efficiently and faster than human embryonic stem cells regardless the site of injection. Stem Cells. 2010 Sep;28(9):1568-70.

16. Nori S, Okada Y, Yasuda A, Grafted humaninduced pluripotent stem-cell-derived neurospheres promote motor functional recovery after spinal cord injury in mice. Proc Natl Acad Sci U S A 2011 Oct 4;108(40):16825-30.

17. Marión RM, Strati K, Li H, et al. A p53-mediated DNA damage response limits reprogramming to ensure iPS cell genomic integrity. Nature 2009 Aug 27;460(7259):1149-53.

18. Luo M, Ling T, Xie W, NuRD blocks reprogramming of mouse somatic cells into pluripotent stem cells. Stem Cells. 2013 Jul;31(7):1278-86.

19. Yoach R, Asaf Z, Shay G, et al. Deterministic direct reprogramming of somatic cells to pluripotency. Nature 2013;502:65-70.

20. Selvaraj V, Plane JM, Williams AJ, Deng W. Switching cell fate: the remarkable rise of induced pluripotent stem cells and lineage reprogramming technologies. Trends Biotechnol 2010 Apr;28(4):214-23.

21. Zhao XY, Li W, Lv Z, Liu L, et al. iPS cells produce viable mice through tetraploid complementation. Nature 2009 Sep 3;461(7260):86-90.

22. Park IH, Arora N, Huo H, et al. Disease-specific induced pluripotent stem cells. Cell 2008 Sep 5;134(5):877-86.

23. Freedman BS, Lam AQ, Sundsbak JL, et al. Reduced ciliary polycystin-2 in induced pluripotent stem cells from polycystic kidney disease patients with PKD1 mutations. J Am Soc Nephrol 2013 Oct;24(10):157186.

24. Baker M. Miniature human liver grown in mice. 3 July 2013. Nature.com. Retrieved 19 July 2013.

25. Kobayashi T, Yamaguchi T, Hamanaka S, et al. Generation of rat pancreas in mouse by interspecific blastocyst injection of pluripotent stem cells. Cell 2010 Sep 3;142(5):787-99.

26. Eiraku M, Watanabe K, Matsuo-Takasaki M, et al. Self-organized formation of polarized cortical tissues from ESCs and its active manipulation by extrinsic signals. Cell Stem Cell 2008 Nov 6;3(5):519-32.

27. Park TS, Bhutto I, Zimmerlin L, et al. Vascular progenitors from cord blood-derived induced pluripotent stem cells possess augmented capacity for regenerating ischemic retinal vasculature. Circulation 2014 Jan 21;129(3):359-72. 
28. Tang $\mathrm{H}$, Sha $\mathrm{H}$, Sun $\mathrm{H}$, et al. Tracking induced pluripotent stem cells-derived neural stem cells in the central nervous system of rats and monkeys. Cell Reprogram 2013 Oct;15(5):435-42.

29. First transfusions of manufactured" blood planned for 2016. Gizmag.com. Retrieved 2014-04-23.

30. Egawa N, Kitaoka S, Tsukita K, et al. Drug screening for ALS using patient-specific induced pluripotent stem cells. Sci Transl Med 2012 Aug 1;4(145):145ra104.

31. Riken Center for Developmental Biology. Information on proposed pilot study of the safety and feasibility of transplantation of autologous hiPSC-derived retinal pigment epithelium (RPE) cell sheets in patients with neovascular age-related macular degeneration. Retrieved 23 July 2013.

32. Gallagher J. Pioneering adult stem cell trial approved by Japan. BBC News. (19 July 2013). Retrieved 23 July 2013.

33. Miura K, Okada Y, Aoi T, et Al. Variation in the safety of induced pluripotent stem cell lines. Nat Biotechnol 2009 Aug;27(8):743-5.
34. Okano H, Nakamura M, Yoshida K, et al. Steps toward safe cell therapy using induced pluripotent stem cells. Circ Res 2013 Feb 1;112(3):523-33.

35. Gerlin A, Roche P. Sanofi Plan \$72.7 Million Stem-Cell Bank. Bloomberg.com. 5 December 2012. Retrieved 23 December 2012.

36. Seki T, Yuasa S, Fukuda K. Generation of induced pluripotent stem cells from a small amount of human peripheral blood using a combination of activated $\mathrm{T}$ cells and Sendai virus. Nat Protoc 2012 Mar 15;7(4):718-28.

37. Staerk J, Dawlaty MM, Gao Q, et al. Reprogramming of human peripheral blood cells to induced pluripotent stem cells. Cell Stem Cell 2010 Jul 2;7(1):20-4.

38. Jurecka A, Zikanova M, Tylki-Szymanska A, et al. Clinical, biochemical and molecular findings in seven Polish patients with adenylosuccinate lyase deficiency. Mol Genet Metab 2008 Aug;94(4):435-42.

39. Loh YH, Hartung O, Li H, et al. Reprogramming of $\mathrm{T}$ cells from human peripheral blood. Cell Stem Cell 2010 Jul 2;7(1):15-9.
Access full text article on other devices

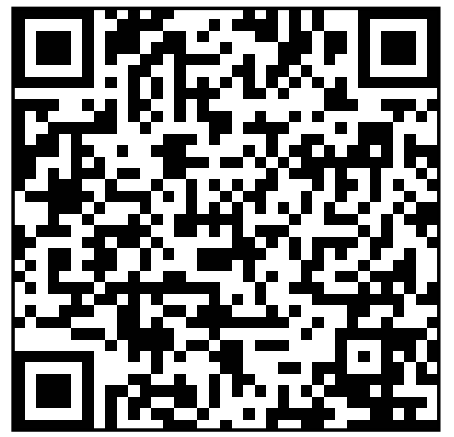

Access PDF of article on other devices

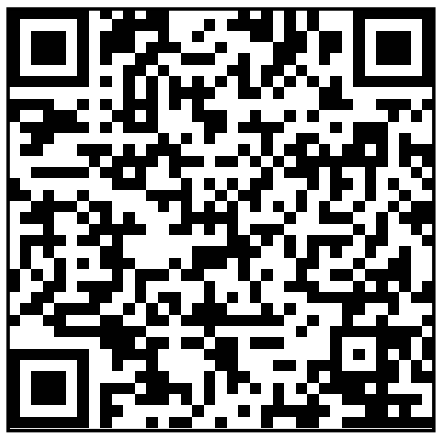

